Bastow, to give an epidural injection of 120 c.c.s of half per cent. procaine in saline under pentothal anaesthesia, followed by gentle rotatary manipulation of the spine, and straight leg raising. A plaster corset is then applied which is worn for three months. At the end of this period, massage and exercises are carried out as above. Should these measures not give relief, and other signs point to the presence of a disc lesion, laminectomy, with removal of the prolapsed nucleus pulposus, is considered.

\title{
REFERENGES
}

BRADFORD, F. K., and SPURLING, R. G. (1941) ' The Intervertebral Disc,' Springfield and Baltimore.

BURT, J. BARNES (1933), Proc. Roy. Soc. Med., 26, 1396. CAPENER (1944), 'Annals of the Rheumatic Diseases,' $4,29$. DENNY BROWN, D. (I933), Proc. Roy. Soc. Med., 26, 1399. DENNY BROWN, D. (I933), Proc. R

ELLIOTT, F. A. (1944), Lancet, 1, 47. (1945), Lancet, $\mathbf{x}, 4$.

HARRIS, W. (1933), Proc. Rov. Soc. Med., 26, 1389.

HOLMES, J. MACD., and SWORN, B. R. (1946), Brit. Med. F.,

I, G46.
LITTLE, N. (1946), Med. Fourn. of Australia, 1, 33. MACEY, H. B. (1940), Arch. Surg., 40, 433.

O'CONNELL, J. E. A. (1946), Brit. Med. Ұ., х, 122.

PUTTI, V. (1927), Lancet, 2, 53. . Surg., 40, 375 .

SPURLIN̈G, R. G., and SCOVILLE, W. B. (1944), Surg. Gynaec. Obstet., 78, 350 .

STEINDLER, A. J. (1940), F. Bone and Foint Surz., 22, 28.

WALSHE, F. M. R. (1946), 'Diseases of the Nervous System,' Edinburgh, E. and S. Livingstone.

\section{THE PLACE OF CAESAREAN SECTION IN MODERN OBSTETRICS}

\author{
By JosePhine Barnes, M.A., D.M., M.R.C.P., F.R.C.S., M.R.C.O.G. \\ Assistant, Obstetric Unit, University College Hospital
}

One of the most striking developments in modern obstetrics has been the immense increase in the number of women delivered by Caesarean section. Two hundred years ago the Caesarean operation was regarded as the last resort in a desperate emergency when delivery was possible by no other means. The mortality was exceedingly high-in fact recovery from the operation was considered very remarkable. Smellie (eighth posthumous edition) wrote concerning the operation that it might be occasionally indicated 'because the mother and child have no other chance to be saved, and it is better to have recourse to an operation which has sometimes succeeded than to leave them both to inevitable death.'

Today the position is very different. It has become necessary for eminent obstetricians to warn their colleagues against the abuse of an operation which, thanks to the development of anaesthesia, of aseptic and antiseptic technique, and to a lesser extent of chemotherapeutic methods has become, in some hands, a means of avoiding even the slightest difficulty in labour. These warnings are timely since no other procedure is more open to abuse. No high degree of operative skill is needed, certainly not for the classical operation, but only wide obstetrical experience can give the judgment needed to decide when delivery by the natural passages is likely to prove sufficiently dangerous to mother or child that operative delivery is to be preferred.

\section{The Advantages of Caesarean Section}

It must not be assumed that because the operation of Caesarean is so easily open to abuse that its wider employment is not, on the whole, a great advance in midwifery. The advantage of reasonably safe abdominal delivery in cases such as those of severely contracted pelvis where natural delivery would be hazardous, if not impossible, are self-evident. The advantages of abdominal delivery may be summed up under two main headings.

Firstly, it avoids the danger of delivery by the vagina and there are many circumstances, as will be seen, where it is inadvisable even to attempt such delivery, while in other cases the course of labour may be seen to be so unfavourable that it seems unwise to allow it to proceed further. Secondly, Caesarean section offers a rapid method of terminating pregnancy and may thus be considered as an alternative to induction of labour. The methods of induction in current use, admirable though they are in favourable cases, are all very uncertain 
in their effect, particularly where the pregnancy is not near term and until some new and more sure means is found, possibly by the endocrinologists, of ensuring the effect of induction, Caesarean section is to be preferred in many cases where a condition affecting mother or child indicates immediate termination of pregnancy.

Here a point of great difficulty often arises, to what extent is it right to submit the mother to Caesarean section in the interest of the child ? In many cases the issue is in no doubt, the risk to the child is great, and there is in addition some risk to the mother if spontaneous delivery is to be attempted. It does seem, however, as though a gradual revolution is taking place in modern obstetric practice and that the value of the life of the child is assuming greater importance even in relation to the safety of the mother. Several factors are contributing to a change in attitude. Later marriage, postponement of childbearing and the tendency to smaller families all tend to increase the value of the child to its parents. It may be argued that this change of attitude on the part of the parents should not influence the judgment of the obstetrician, but there are occasions when it is realized that the loss of the child will devastate the lives of its parents to such an extent, and that the chances of the mother bearing further children is slight, on which may be justified to submit the mother to a slightly increased risk.

\section{The Disadvantages of Caesarean Section}

The most obvious disadvantage of Caesarean section is the increased risk to the mother. Several series of 100 or more cases without a maternal death have been published, but it must be remembered that the average maternal mortality from Caesarean section is not, in the best hands, more than one or two per cent., and that therefore a very large series of cases, probably more than a single operator could or should perform in a lifetime, is required to obtain statistical comparison with the results of spontaneous labour. When it comes to the graver obstetrical complications as, for example, the major degrees of placenta praevia, the maternal mortality from Caesarean section compares favourably with that from other methods of treatment.
Apart from the increased maternal mortality, Caesarean section has other disadvantages to the 'mother. Apart from obvious disabilities which may arise out of complications after the operation, recovery after childbirth is definitely delayed and patients may not fully recover for many months. The patient has the strain of recovering from a major abdominal operation and is at the same time plunged into the arduous routine involved in the care of the infant.

The prognosis for future childbearing depends on many factors. It is unlikely that Caesarean section leads to any diminution in fertility. In a subsequent pregnancy, the chief risk is of rupture of the uterine scar. This risk will depend on the type of operation, being many times higher after classical section than after the lower segment operation. It will depend also on the presence or absence of post-operative infection and to some extent on the indication for which the operation was performed, clearly in a case of contracted pelvis, the risk of rupture will be greater than if the pelvis is normal. But few will now agree with the dictum 'once a Caesarean always a Caesarean.' In a recent paper from the United States, a large number of women who had had lower segment Caesarean section were followed through a subsequent pregnancy and labour-like normal primigravidae, and that in the absence of any, recurring cause the risk to mother or child was not affected by the previous operation. In spite of these reassuring figures, the conduct of labour in a patient who has undergone abdominal section is likely to be more anxious than in one who has already had a delivery by the natural passages.

Does Caesarean section offer greater safety to the foetus? In theory it should do so, but in practice it is found that there is a minimal foetal mortality even in the best hands and many obstetricians of experience believe that the foetus gains something from normal labour. A great deal depends, of course, on the condition necessitating section, on the skill of the operator and on the anaesthetic. With the premature baby, foetal mortality is high, but here it is probably because the infants are premature rather than from any effect of the operation, though a premature infant is 
naturally more susceptible to the effects of inhalation anaesthesia administered to the mother.

One of the worst tragedies in obstetrics is to perform a Caesarean section perhaps for the sake of the child and then to find that the infant is seriously deformed. The particular deformity likely to cause such an error to be made is hydrocephalus. Before performing Caesarean section in a case of disproportion the possibility of an abnormal foetus should always be borne in mind and every possible step taken to exclude this.

In summing up the general remarks on the place of Caesarean section in modern obstetrics one may say that the enormous increase in the performance of the operation has, on the whole, been of, benefit to mother and child, that Caesarean section is now being performed more often in the interest of the child, that an unnecessary Caesarean is a major error of obstetrical judgment and it is one which may lead to tragedy or may prejudice the chances of the mother in future pregnancies.

\section{Indications for Caesarean Section}

It is usual to divide the indications for Caesarean section into relative and absolute. Absolute indications are a contracted pelvis with, in this country, a true conjugate of less than $3 \frac{1}{2}$ in., and a pelvic tumour that is causing insuperable obstruction to delivery. Relative indications now include a wide variety of conditions, and the decision for or against section often depends on a number of considerations including the age of the mother, her previous obstetrical history, and period of sterility and the severity of the condition for which section is contemplated. The primigravida over 40 years of age needs special consideration since it is known that such patients are liable to complications of all kinds, that the incidence of toxaemia is high, and that instrumental delivery is often necessaty. Such a patient may have little chance of bearing more children, and though it is not suggested that Caesarean section should always be performed, any relatively slight abnormality, such as a high head or uterine inertia should lead the obstetrician to favour operation rather than risk perhaps a long labour with a difficult delivery and even a stillborn child at the end of it.
Of the many relative indications for Caesarean section, three groups merit special consideration :-contracted pelvis, antepartum haemorrhage and toxaemia of late pregnancy.

\section{Caesarean Section for Contracted Pelvis}

The treatment of contracted pelvis will be dealt with more fully in a subsequent article on the subject of difficult labour. The modern position with regard to contracted pelvis seems to be that most obstetricians in this country agree that with a true conjugate of less than $3 \frac{1}{2}$ in. delivery of a normal infant per vaginam is unlikely, or may be fraught with great difficulty. In such cases elective Caesarean section a week or so before term is indicated. For the case where the conjugate exceeds $3 \frac{1}{2}$ in. but is less than $4 \frac{1}{2}$ in. the modern tendency is to choose trial of labour for the young primigravida, provided always that the vertex presents. In elderly primigravida and in cases of breech presentation where prophylactic external version has failed, Caesarean section is to be deferred. It is important to emphasize the value of $\mathrm{X}$-ray pelvimetry in cases where the size or shape of the pelvis is in doubt.

\section{Caesarean Section for Antepartum Haemorrhage}

It is now generally recognized that antepartum haemorrhage, however slight, should be regarded as one of the gravest of obstetrical emergencies. When haemorrhage, however slight, occurs after the 28 th week of pregnancy, the patient should be immediately removed to an institution with facilities for immediate Caesarean section and blood transfusion, and for such other methods of treatment as may be required. The cervix and vagina are inspected with a sterile speculum to exclude any extraplacental cause for bleeding. If none is found, it is our practice to do nothing further for the patient, except to keep her under observation in hospital until term. The indication to act is severe haemorrhage and if this occurs the patient is examined per vaginam in an operating theatre with preparations for Caesarean section and other forms of treatment. If a placenta praevia is found, Caesarean section should be performed immediately in all cases where the 
placenta covers the internal os or is within easy reach of it. It may also be preferred in an elderly primigravida with a more minor degree of placenta praevia. We cannot agree with the practice of performing Caesarean section in all cases of antepartum haemorrhage, without first attempting to determine the cause. Interference, including vaginal examination is contraindicated in cases where the haemorrhage is only slight, and the examination may provoke severe bleeding or cause the onset of premature labour, to say nothing of the risk of introducing infection. A minor degree of placenta praevia can often be successfully treated by the more conservative method of rupturing the membranes, but in the major degrees Caesarean section offers the best chance for both mother and child. From the opinions expressed at a recent meeting of the Section of Obstetrics and Gynaecology of the Royal Society of Medicine, it is clear that modern obstetric opinion has swung strongly in favour of Caesarean section for placenta praevia, combined with expectant treatment for the initial haemorrhage to give the child the chance of reaching sufficient maturity for survival.

In accidental antepartum haemorrhage, Caesarean section may occasionally be indicated. It is the best treatment for the unusual case of severe external haemorrhage with a. living baby and it may be indicated in the rare cases of concealed haemorrhage which do not respond to conservative treatment. The majority of these cases, however, are best treated by other means.

\section{Caesarean Section for Toxaemia of Late Pregnancy}

In cases of severe pre-eclamptic toxaemia there are three main dangers, there may be eclampsia, the child may die in utero or accidental haemorrhage may occur. Most cases can be treated expectantly but in those cases where there is deterioration in spite of such treatment, with rising blood pressure and increase in albuminuria, termination of pregnancy may be desirable. In many cases, especially of course in the elderly primigravida, Caesarean section may be preferred to induction, and particularly in cases where there is sudden deterioration. The operation is a rapid and certain way of terminating pregnancy, whereas induction, especially when the pregnancy is not at term, is often uncertain and some time may elapse before delivery. Section should be deferred if possible until the end of the $37^{\text {th }}$ week to give the child the best chance of survival. Similarly in the preeclamptic state section may be performed after a preliminary brief period of medical treatment including sedatives, starvation and venesection. Eclampsia occurring before labour is generally treated expectantly by a modification of the Stroganoff method, but if after the convulsions have been controlled, the foetus in utero is followed by marked improvement in maternal symptoms and signs, these cases can usually be left to deliver themselves.

Caesarean section may also be performed in two other varieties of toxaemia of late pregnancy, hypertensive toxaemia (chronic nephritis in pregnancy), where there is great liability for the foetus to die in utero during the last month of pregnancy. Unless urgent symptoms appear it is advisable to wait until the end of the $37^{\text {th }}$ week as these mothers tend to have small infants.

It is not suggested that Caesarean section is to be considered in any way as a routine procedure for cases of toxaemia of late pregnancy. As with other indications for section, the needs of each particular case needs to be carefully weighed before the decision.

\section{Miscellaneous Indications for Caesarean Section}

It is not proposed to give a list of all the many and varied conditions for which Caesarean section may on occasion be indicated but rather to indicate certain fairly common conditions which are often best dealt with by this means.

The presence of fibroids in the pregnant uterus is often considered to be an indication for section, but it is doubtful whether this should be a general rule. A fibroid impacted in the pelvis and obstructing labour should be dealt with by abdominal section, but this is a relatively rare finding and many patients even with large fibromata deliver themselves spontaneously. The chief troubles caused by fibroids are malpresentation and uterine inertia. Many cases can be given a short trial of 
labour, which can be terminated by abdominal section if all is not going well, particularly if the membranes rupture prematurely or if inertia appears. Caesarean section seems to be performed too readily for fibroids.

Persistent malpresentation may indicate Caesarean section, especially in a primigravida, and provided there is no evidence of foetal abnormality. This applies to cases of breech presentation where there is some complication such as even a slight degree of pelvic contraction. Prolapse. of the cord occurring early in labour may be another indication, provided the foetus is alive. Attempts to replace the cord and keep. it in position are often unsuccessful, and the manipulations necessary may tend to lead to uterine infection.

In conditions where death of the foetus in utero tends to occur in the later weeks of pregnancy, Caesarean section may be performed in the interest of the child. This has already been mentioned in relation to toxaemia of late pregnancy. Maternal diabetes is considered by some to be such an indication, though in a multipara induction of premature labour may be preferred. Habitual death of the foetus in utero, an obscure and not common condition may also indicate section. It is probable that some cases of foetal erythroblastosis may be saved by section, provided the condition has not progressed too far before the child is viable. In a rhesus negative mother samples of maternal blood should be tested regularly for antibodies and if these appear or are increasing, operation may be performed to remove the child; it is viable from the unfavourable environment. Facilities should be available for transfusing the child with rhesus negative blood if required. It is difficult to be dogmatic about a condition concerning which knowledge is accumulating almost daily and a few years should see the position greatly clarified. In the light of present knowledge though it does seem that timely intervention may save some infants that would otherwise be lost.

\section{Technique}

Modern obstetric opinion is tending to favour the lower segment operation for most cases where abdominal section is indicated. This procedure has many advantages. There is generally less haemorrhage, less risk: of general peritonitis, a smoother convalescence and, above all, a much diminished risk of rupture of the uterine scar in a subsequent pregnancy and labour than with the classical operation. For the infected case, the lower segment operation gives a much lower maternal mortality and morbidity rate. In these cases some operators now prefer the extraperitoneal operation, avoiding entirely opening the peritoneal cavity. The technique of this operation is more difficult than that of the introperitoneal operations, but excellent results are claimed from its use.

The question of anaesthesia for Caesarean section is one which raises much controversy. In this country, local analgesia, at least until the baby is born, has many protagonists. It has many advantages for both mother and child, haemorrhage is usually slight and the condition of the infant excellent. It seems especially advantageous for the premature infant which is very susceptible to inhalation anaesthesia given to the mother. Spinal, caudal and the various types of inhalation anaesthesia all have their supporters ant opponents, and a great deal will depend op conditions and on the skill and experience of the operator and the anaesthetist.

\section{Conclusions}

In this article an attempt has been made to survey the place of Caesarean section in modern obstetrics and to indicate the types of cases where the operation is advantageous and where its performace represents a real advance on older methods of treatment. It must end with a further warning against abuse of the operation which is so easily decided upon, so easily performed and yet which may, on occasion, lead to unexpected tragedy. Truly the skill lies not in the performance of the operation, but in deciding when it is to be performed.

\section{BIBLIOGRAPHY}

In this article the various topics have been dealt with along general lines and no detailed list of references to papers will be given. The following works have been widely consulted and are given. The following works have been widely consul
recommended to those who wish to read further :-

BROWNE, F. J. (1946), "Antenatal and Postnatal Care," 6th Edition, Churchill, London.

MARSHALL, C. McINTOSH (1939), "Caesarean Section-the Lower Segment Operation," Wright, Bristol.

KERR, J. M. MUNRO (1937), "Operative Obstetrics," 4t Edition, Balliere, Tindall and Cox, London.

YOUNG, J. H. (1944), "The History of Caesarean Section," H. K. Lewis, London. 